\title{
PENALTY METHOD FOR REFLECTED DIFFUSIONS ON THE HALF-LINE
}

\author{
CAMERON BRUGGEMAN AND ANDREY SARANTSEV
}

\begin{abstract}
Consider a reflected diffusion on the positive half-line. We approximate it by solutions of stochastic differential equations using the penalty method: We emulate the "hard barrier" of reflection by a "soft barrier" of a large drift coefficient, which compells the diffusion to return to the positive half-line. The main tool of the proof is convergence of scale functions.
\end{abstract}

\section{INTRODUCTION}

Let us informally introduce the concept of a reflected diffusion process on the positive half-line $\mathbb{R}_{+}:=[0, \infty)$. Take measurable functions $g: \mathbb{R}_{+} \rightarrow \mathbb{R}$ and $\sigma: \mathbb{R}_{+} \rightarrow \mathbb{R}_{+}$. An $\mathbb{R}_{+}$-valued stochastic process $Z=(Z(t), t \geq 0)$ is called a reflected diffusion on $\mathbb{R}_{+}$with drift coefficient $g$ and diffusion coefficient $\sigma$ if:

(i) as long as $Z(t)>0$, this process behaves as a solution to the following SDE:

$$
\mathrm{d} Z(t)=g(Z(t)) \mathrm{d} t+\sigma(Z(t)) \mathrm{d} W(t),
$$

where $W=(W(t), t \geq 0)$ is a Brownian motion on the real line;

(ii) at $z=0$, it is reflected in the positive direction.

The precise definition is given in Section 2. By construction, this process $Z$ cannot assume negative values. In other words, it cannot penetrate "the barrier at zero". This article is devoted to the following question: can we "approximate" this "hard barrier" by a "soft barrier" created by a large drift coefficient? More specifically, consider the solution to the following SDE:

$$
\mathrm{d} X(t)=f(X(t)) \mathrm{d} t+\tilde{\sigma}(X(t)) \mathrm{d} W(t), \quad X(0)=Z(0) .
$$

Assume that $f(z) \approx g(z)$ for positive $z$ away from zero, $f(z)$ is positive and very large when $z \approx 0$, and $\tilde{\sigma}(z) \approx \sigma(z)$ for all $z \geq 0$. Then we can hope that $X \approx Z$ in distribution. A precise statement of this result is given in Theorem 2.1.

Let us give a simple example. Suppose we wish to approximate reflected Brownian motion $Z=(Z(t), t \geq 0)$ on the positive half-line by a solution of SDE. Assume $Z(0)=z>0$. Consider the following sequence of diffusions:

$$
\mathrm{d} X_{n}(t)=a_{n} 1_{\left[-c_{n}, 0\right]}\left(X_{n}(t)\right) \mathrm{d} t+\mathrm{d} W(t), \quad X_{n}(0)=z .
$$

where $a_{n}, c_{n}>0$ are such that

$$
a_{n} \rightarrow \infty, a_{n} c_{n} \rightarrow \infty
$$

Then for every $T>0$, the law of $X_{n}$ on the space $C[0, T]$ of continuous real-valued functions on $[0, T]$ weakly converges to the law of $Z$ on the same space.

Date: October 12, 2016. Version 16.

2010 Mathematics Subject Classification. Primary 60J60, secondary 60J55, 60J65, 60H10.

Key words and phrases. Stochastic differential equation, reflected diffusion, reflected Brownian motion, weak convergence, scale function, penalty method. 
In the literature, this is sometimes called the penalty method, with the penalty function $f(x)-$ $g(x)$. For example, in the setting (11), the penalty function is $a_{n} 1_{\left[-c_{n}, 0\right]}(x)$. This method aims to emulate the reflection by a strong drift which is directed inside the domain of reflection. Our companion paper [25] deals with the case of multidimensional reflected Brownian motion in a domain $D \subseteq \mathbb{R}^{d}$, including the case of oblique (not normal) reflection.

The main idea of the proof is to consider scale functions:

$$
s_{n}(x)=\int_{c_{n}}^{x} \exp \left(-2 \int_{b_{n}}^{y} \frac{f_{n}(z)}{\sigma_{n}^{2}(z)} \mathrm{d} z\right) \mathrm{d} y .
$$

The integration limits $c_{n}$ and $b_{n}$ of integration can be chosen arbitrarily, because the function $s_{n}$ is defined up to an additive and a multiplicative constant. With a certain choice of $s_{n}$, we have:

$$
s_{n}(x) \rightarrow\left\{\begin{array}{l}
s(x), x>0 ; \\
-\infty, x<0,
\end{array} \quad \text { where } s(x):=\int_{0}^{x} \exp \left(-2 \int_{0}^{y} \frac{g(z)}{\sigma^{2}(z)} \mathrm{d} z\right) \mathrm{d} y\right.
$$

can be viewed as the scale function for the reflected diffusion $Z$.

1.1. Historical review. Reflected diffusions in one or many dimensions were studied extensively since the 1960s. The concept of reflected diffusion was pioneered by Skorohod in the papers [27, 28]; see also the book [6], and an article [17]. Without attempting to conduct an extensive survey, let us mention the following articles on multidimensional reflected Brownian motion in general regions: [7, 8, 9, 14, 30, 31, 32, 33, 34].

Now, let us survey the literature on the penalty method. The papers [21, 35] apply the theory of Dirichlet forms to use penalty method for the case of stationary processes. A related paper [20] also deals with stationary distributions for the penalized Brownian motion in a convex polyhedron, which is intended to approximate a semimartingale reflected Brownian motion in this polyhedron; the authors call this soft reflection. The paper [29] applies the penalty method to a multidimensional reflected diffusion in a convex domain $D$, with the penalty function $x \mapsto n(x-\Pi(x))$; here, $\Pi(x)$ is the projection of $x$ on $D$. In the companion paper [25] mentioned above, we generalize this approach to more general penalty functions. The papers [11, 12, 18, 19] apply a similar technique to stochastic differential equations with jumps; see also a related paper [16]. The penalty method with penalty function $x \mapsto n x_{-}$was also used in [4] to approximate reflected backward stochastic differential equations (BSDE) on the real line with non-reflected BSDE and to prove existence of a solution of a reflected BSDE. In the mutlidimensional setting, similar work was carried out in [10, 24]. Finally, let us mention the articles [1, 3, 5, 14, 15, 26] on the penalty method.

1.2. Relation with skew Brownian motion. A survey [13] contains a few ways to construct a skew Brownian motion. This can be loosely described as a Brownian motion such that each excursion is independently filpped to the positive half-line with a certain probability $\alpha \in(0,1)$, and to the negative half-line with probability $1-\alpha$. Here, $\alpha$ is called the skewness parameter. This process is, in some sense, between a usual Brownian motion and a reflected Brownian motion: for $\alpha=1 / 2$ we get the usual Brownian motion, and for $\alpha=1$ we get a reflected Brownian motion.

The survey [13] contains some results about approximation of a skew Brownian motion by solutions of SDEs. In the setting of (1), if $a_{n} c_{n} \rightarrow k>0$ as $n \rightarrow \infty$ instead of $a_{n} c_{n} \rightarrow \infty$, then $X_{n}$ converges in law to a skew Brownian motion with the skewness parameter

$$
\alpha(k):=\frac{e^{2 k}}{1+e^{2 k}} .
$$

For $k \rightarrow \infty$, we have: $\alpha(k) \rightarrow 1$. Therefore, one expects the skew Brownian motion to converge in law to the reflected Brownian motion. This is a natural way to arrive at the conditions (2). Related papers [22, 23] deal with multidimensional skew Brownian motions. 
1.3. Organization of the paper. Section 2 is devoted to the setup of the problem and the main result (Theorem 2.1), as well as corollaries and examples (including the one mentioned above). The proof of Theorem 2.1 is given in Section 3. The Appendix contains some technical lemmata.

\section{Definitions And the Main Result}

2.1. Notation. Denote weak convergence by the arrow $\Rightarrow$. Let $C[0, T]$ be the space of continuous functions $[0, T] \rightarrow \mathbb{R}$. We let $\inf \varnothing=\infty$. A standard Brownian motion is a one-dimensional Brownian motion, starting from zero, with zero drift coefficient and unit diffusion coefficient. For an event $A$, let $A^{c}$ be its complement. Take a continuous function $f:[a, b] \rightarrow \mathbb{R}$. For $\delta>0$, its modulus of continuity corresponding to $\delta$ is denoted as follows:

$$
\omega(f,[a, b], \delta):=\max _{\substack{t_{1}, t_{2} \in[a, b] \\\left|t_{1}-t_{2}\right| \leq \delta}}\left|f\left(t_{1}\right)-f\left(t_{2}\right)\right| .
$$

2.2. The concept of a reflected diffusion. We operate in a standard setting: a filtered probability space $\left(\Omega, \mathcal{F},\left(\mathcal{F}_{t}\right)_{t \geq 0}, \mathbf{P}\right)$, with the filtration $\left(\mathcal{F}_{t}\right)_{t \geq 0}$ satisfying the usual conditions. Take a standard $\left(\mathcal{F}_{t}\right)_{t \geq 0}$-Brownian motion $W=(W(t), t \geq 0)$. Consider two measurable functions $g: \mathbb{R}_{+} \rightarrow \mathbb{R}$ and $\sigma: \mathbb{R}_{+} \rightarrow \mathbb{R}_{+}$.

Definition 1. Take a continuous adapted process $Z=(Z(t), t \geq 0)$ with values in $\mathbb{R}_{+}$, as well as another continuous adapted process $L=(L(t), t \geq 0)$, with the following properties:

(i) $L(0)=0, L$ is nondecreasing and can increase only when $Z=0$; we can write the last property formally as

$$
\int_{0}^{\infty} Z(t) \mathrm{d} L(t)=0
$$

(ii) for all $t \geq 0$,

$$
Z(t)=Z(0)+\int_{0}^{t} g(Z(s)) \mathrm{d} s+\int_{0}^{t} \sigma(Z(s)) \mathrm{d} W(s)+L(t)
$$

Then the process $Z$ is called a reflected diffusion on $\mathbb{R}_{+}$with drift coefficient $g$ and diffusion coefficient $\sigma$. The process $L$ is called the reflection term corresponding to $Z$. If $Z(0)=z_{0}$, we say $Z$ starts from $z_{0}$.

Remark 1 . The differential $\mathrm{d} L$ can be viewed as "the push" which does not allow $Z$ to go negative when $Z$ is at zero.

There are many conditions on the coefficients $g$ and $\sigma^{2}$ which ensure the weak or strong existence and uniqueness of a reflected diffusion together with the corresponding reflection term, [27, 28, 17]. We will just assume that weak existence and uniqueness in law hold. For the rest of the article, we fix $z_{0}>0$.

Assumption 1. For this fixed $z_{0}>0$, the process $Z$ described in Definition 1 exists in the weak sense and is unique in law.

To state the main result, we need some additional conditions on $g$ and $\sigma$.

Assumption 2. The functions $g$ and $\sigma$ are continuous on $\mathbb{R}_{+}$, and $\sigma(x)>0$ for all $x \geq 0$. 
2.3. Main result. Let again $W=(W(t), t \geq 0)$ be a standard $\left(\mathcal{F}_{t}\right)_{t \geq 0}$-Brownian motion. Take two sequences $\left(f_{n}\right)_{n \geq 1}$ and $\left(\sigma_{n}\right)_{n \geq 1}$ of measurable functions

$$
f_{n}: \mathbb{R} \rightarrow \mathbb{R}, \quad \sigma_{n}: \mathbb{R} \rightarrow \mathbb{R}_{+}, \quad n=1,2, \ldots
$$

Consider a sequence $\left(X_{n}\right)_{n \geq 1}$ of approximating diffusions:

$$
\mathrm{d} X_{n}(t)=f_{n}\left(X_{n}(t)\right) \mathrm{d} t+\sigma_{n}\left(X_{n}(t)\right) \mathrm{d} W(t), X_{n}(0)=z_{n}
$$

Assumption 3. For each $n=1,2, \ldots$, the coefficients $f_{n}, \sigma_{n}$ and the initial condition $z_{n}$ are such that the process $X_{n}$ defined in (3) exists in the weak sense on the infinite time horizon and is unique in law.

Let us also make an assumption about the initial conditions. We decided to state this separately from the conditions in the body of Theorem [2.1, which deal with $f_{n}$ and $\sigma_{n}$.

Assumption 4. $z_{n} \rightarrow z_{0}$ as $n \rightarrow \infty$.

Now, let us state the main result of the paper.

Theorem 2.1. Under Assumptions 1 - 4, suppose that:

(i) there exists $\varepsilon_{0}>0$ such that for every $\varepsilon \in\left(0, \varepsilon_{0}\right)$,

$$
\lim _{n \rightarrow \infty} \int_{-\varepsilon}^{\varepsilon} f_{n}(x) \mathrm{d} x=\infty
$$

(ii) for every $\left[x_{1}, x_{2}\right] \subseteq(0, \infty)$,

$$
\int_{x_{1}}^{x_{2}}\left|f_{n}(x)-g(x)\right| \mathrm{d} x \rightarrow 0, \quad n \rightarrow \infty
$$

(iii) for every $x>0$, we have: $\sigma_{n} \rightarrow \sigma$ uniformly on $[0, x]$;

(iv) the family of functions $\left(\sigma_{n}\right)_{n \geq 1}$ is equicontinuous at $x=0$, that is,

$$
\limsup _{\delta \rightarrow 0} \sup _{n \geq 1}\left|\sigma_{n}(x)-\sigma_{n}(0)\right|=0 .
$$

(v) for every $\varepsilon>0$, there exist $n_{0}$ and $\delta>0$ such that

$$
f_{n}(x)>g(x)-\varepsilon \text { for } n>n_{0},|x|<\delta .
$$

Then for every $T>0$, as $n \rightarrow \infty$,

$$
X_{n} \Rightarrow Z \text { weakly in } C[0, T] \text {. }
$$

2.4. The case of reflected Brownian motion. Consider a special case, when $Z$ is a reflected Brownian motion on $\mathbb{R}_{+}$, that is, $g \equiv 0$ and $\sigma \equiv 1$. Suppose we decide to approximate it by diffusion processes with diffusion coefficients 1 , that is, $\sigma_{n} \equiv 1, n=1,2, \ldots$ Then the statement of the theorem can be simplified a bit.

Corollary 2.2. Under Assumption 3, suppose that:

(i) there exists $\varepsilon_{0}>0$ such that for every $\varepsilon \in\left(0, \varepsilon_{0}\right)$, we have:

$$
\lim _{n \rightarrow \infty} \int_{-\varepsilon}^{\varepsilon} f_{n}(x) \mathrm{d} x=\infty
$$

(ii) for every $\left[x_{1}, x_{2}\right] \subseteq(0, \infty)$, we have:

$$
\lim _{n \rightarrow \infty} \int_{x_{1}}^{x_{2}}\left|f_{n}(x)\right| \mathrm{d} x=0
$$


(iii) for every $\varepsilon>0$, there exist $n_{0}$ and $\delta>0$ such that

$$
f_{n}(x)>-\varepsilon \text { for } n>n_{0},|x|<\delta \text {. }
$$

Then the diffusion processes $X_{n}$ defined in (3) converge weakly to a reflected Brownian motion on $\mathbb{R}_{+}$in $C[0, T]$, for every $T>0$.

2.5. Examples. Now, let us present a few applications of Corollary 2.2, starting with the example in the Introduction.

Example 1. Take two sequences $\left(a_{n}\right)_{n \geq 1}$ and $\left(c_{n}\right)_{n \geq 1}$ of positive numbers, and let

$$
f_{n}(x)=a_{n} 1_{\left(-c_{n}, 0\right)}(x) .
$$

Then the sequence $\left(f_{n}\right)_{n \geq 1}$ always satisfies conditions (ii) and (iii) of Corollary 2.2, It is an easy exercise to see that condition (i) is satisfied if and only if

$$
\lim _{n \rightarrow \infty} a_{n}=\infty, \lim _{n \rightarrow \infty} a_{n} c_{n}=\infty .
$$

Example 2. Let us "erect the wall" as in previous example, but to the right of $x=0$ : take two sequences $\left(a_{n}\right)_{n \geq 1}$ and $\left(c_{n}\right)_{n \geq 1}$ of positive numbers, and let

$$
f_{n}(x)=a_{n} 1_{\left(0, c_{n}\right)}(x) .
$$

Once again, this satisfies the conditions of Corollary 2.2 if and only if:

$$
\lim _{n \rightarrow \infty} a_{n}=\infty, \quad \lim _{n \rightarrow \infty} c_{n}=0, \text { and } \lim _{n \rightarrow \infty} a_{n} c_{n}=\infty .
$$

Example 3. Take a measurable function $\psi: \mathbb{R} \rightarrow \mathbb{R}$ with the following properties:

$$
\varliminf_{x \rightarrow 0} \psi(x) \geq 0 ; \quad \lim _{y \rightarrow \infty} \int_{-y}^{y} \psi(x) \mathrm{d} x=: I \in(0, \infty) ; \quad \lim _{x \rightarrow \infty} \psi(x)=0 .
$$

Take two sequences $\left(a_{n}\right)_{n \geq 1}$ and $\left(c_{n}\right)_{n \geq 1}$ of positive real numbers which satisfy (5) and, in addition,

$$
\lim _{n \rightarrow \infty}\left[a_{n} \sup _{x \geq x_{0} / c_{n}}|\psi(x)|\right]=0 \text { for every } x_{0}>0 .
$$

Define the following sequence of functions:

$$
f_{n}(x):=a_{n} \psi\left(x / c_{n}\right), \quad n=1,2, \ldots
$$

Then this sequence of functions satisfies the conditions of Corollary 2.2. Indeed, let us verify condition (i): for every $\varepsilon>0$, as $n \rightarrow \infty$,

$$
\int_{-\varepsilon}^{\varepsilon} f_{n}(x) \mathrm{d} x=a_{n} c_{n} \int_{-\varepsilon / c_{n}}^{\varepsilon / c_{n}} \psi(y) \mathrm{d} y \rightarrow \infty,
$$

because from (5) and (6) we get:

$$
a_{n} c_{n} \rightarrow \infty \text { and } \int_{-\varepsilon / c_{n}}^{\varepsilon / c_{n}} \psi(y) \mathrm{d} y \rightarrow I>0 .
$$

Next, condition (ii) follows from (7), and condition (iii) follows from $\underline{\lim }_{x \rightarrow 0} \psi(x) \geq 0$. Examples 1 and 2 are actually two particular cases of this more general example, with

$$
\psi(x)=1_{[-1,0]}(x) \text { and } \psi(x)=1_{[0,1]}(x),
$$


correspondingly. Note that (7) follows automatically from (5) when $\psi$ has compact support, as in (8). There are other examples of functions $\psi$ which satisfy (66), such as $\psi(x)=e^{-|x|} 1_{(-\infty, 0]}$. This latter function was used to construct the so-called exponential reflected Brownian motion in the paper [20]. We consider this topic in our companion paper [25].

\section{Proof of Theorem 2.1}

3.1. Outline of the proof. For the rest of this section, we fix the time horizon $T>0$. Let us split the proof of the main result, Theorem [2.1, into a few lemmata. Every subsection of this section will contain a proof of the corresponding lemma. In the current subsection, we enunciate these lemmata and show how they fit together.

Extend the continuous functions $g, \sigma: \mathbb{R}_{+} \rightarrow \mathbb{R}$ to the whole real line by making them constant on the negative half-line: $g(x):=g(0)$ and $\sigma(x):=\sigma(0)$ for $x \leq 0$. First, we localize: namely, we stop processes $X_{n}$ when they hit a certain level $C>0$. More formally, for every $C>0$, let

$$
T_{C}^{(n)}:=\inf \left\{t \in[0, T] \mid X_{n}(t)=C\right\} \wedge T, \quad n=1,2, \ldots
$$

Denote the stopped processes by

$$
X_{n}^{C}(t) \equiv X_{n}\left(t \wedge T_{C}^{(n)}\right) .
$$

Lemma 3.1. Suppose we proved that for every $C>0$, every weak limit point of $\left(X_{n}^{C}\right)_{n \geq 1}$ in $C[0, T]$ behaves as the reflected diffusion $Z$ until it hits $C$. Then $X_{n} \Rightarrow Z$ in $C[0, T]$.

Remark 2. Note that we are not concerned with behavior of this weak limit point after it hits $C$. It might be stopped there, or it might still move after this hitting moment. We only need this weak limit point to behave as the reflected diffusion $Z$ before it hits $C$.

Now, fix $C>0$. The next part of the proof is devoted to showing that for large $n$, the process $X_{n}^{C}$ does not get far away into the negative half-line $(-\infty, 0)$.

Lemma 3.2. For every $\varepsilon>0$,

$$
\lim _{n \rightarrow \infty} \mathbf{P}\left(\min _{0 \leq t \leq T} X_{n}^{C}(t) \leq-\varepsilon\right)=0 .
$$

For $n=1,2, \ldots$ and $t \in[0, T]$, denote

$$
\begin{gathered}
L_{n}(t)=\int_{0}^{t \wedge T_{C}^{(n)}}\left[f_{n}\left(X_{n}(u)\right)-g\left(X_{n}(u)\right)\right] \mathrm{d} u, \\
Z_{n}(t)=X_{n}^{C}(t)-L_{n}(t)=z_{n}+\int_{0}^{t \wedge T_{C}^{(n)}} g\left(X_{n}^{C}(u)\right) \mathrm{d} u+\int_{0}^{t \wedge T_{C}^{(n)}} \sigma_{n}\left(X_{n}(u)\right) \mathrm{d} W(u) .
\end{gathered}
$$

Lemma 3.3. The sequence $\left(X_{n}^{C}\right)_{n \geq 1}$ is tight in $C[0, T]$.

Fix an increasing sequence $\left(n_{k}\right)_{k \geq 1}$ of positive integers.

Lemma 3.4. We can find a subsequence $\left(n_{k}^{\prime}\right)_{k \geq 1}$ of $\left(n_{k}\right)_{k \geq 1}$ such that, after changing the probability space, we have the following a.s. convergence in $C[0, T] \times C[0, T]$ :

$$
\left(X_{n_{k}^{\prime}}^{C}, Z_{n_{k}^{\prime}}\right) \rightarrow(\bar{Z}, \tilde{Z}), \quad k \rightarrow \infty
$$

The limiting processes $\bar{Z}$ and $\tilde{Z}$ satisfy the following relation: for

$$
t<T_{C}^{\bar{Z}}:=\inf \{t \in[0, T] \mid \bar{Z}(t)=C\} \wedge T
$$


we have:

$$
\tilde{Z}(t)=z_{0}+\int_{0}^{t} g(\bar{Z}(u)) \mathrm{d} u+\int_{0}^{t} \sigma(\bar{Z}(u)) \mathrm{d} \bar{W}(u),
$$

where $\bar{W}=(\bar{W}(u), u \geq 0)$ is a certain standard Brownian motion.

Now, a.s. uniformly on $[0, T]$, as $k \rightarrow \infty$,

$$
L_{n_{k}^{\prime}} \equiv X_{n_{k}^{\prime}}^{C}-Z_{n_{k}^{\prime}} \rightarrow L:=\bar{Z}-\tilde{Z}
$$

Since $L_{n_{k}^{\prime}}(0)=0$ for all $k=1,2, \ldots$, we have: $L(0)=0$.

Lemma 3.5. On the interval $\left[0, T_{C}^{\bar{Z}}\right]$, the process $\bar{L}$ is a.s. nondecreasing, and it can increase only when $\bar{Z}=0$.

Combining the results of Lemmata 3.4, and 3.5, we get the following result: The process $\bar{Z}$ is a version of the reflected diffusion on $\mathbb{R}_{+}$with drift coefficient $g$ and diffusion coefficient $\sigma$, starting from $z$, at least until it hits the level $C$. We arrive at the conclusion that for every subsequence $\left(n_{k}\right)_{k \geq 1}$ there exists a subsequence $\left(n_{k}^{\prime}\right)_{k \geq 1}$ such that $X_{n_{k}^{\prime}}^{C}$ weakly converges (in $C[0, T]$, as $k \rightarrow \infty$ ) to a process $\bar{Z}$ is a version of this reflected diffusion $Z$, at least until $\bar{Z}$ hits $C$. Use Lemma 3.1 to complete the proof of Theorem 2.1.

3.2. Proof of Lemma 3.1. Take a small probability $\eta>0$. Then there exists $C>0$ such that

$$
\mathbf{P}\left(\max _{0 \leq t \leq T} Z(t)<C\right) \geq 1-\eta \text {. }
$$

Fix an increasing sequence $\left(n_{k}\right)_{k \geq 1}$ of positive integers. Then it has a subsequence $\left(n_{k}^{\prime}\right)_{k \geq 1}$ such that , in $C[0, T]$,

$$
X_{n_{k}^{\prime}}^{C} \Rightarrow \bar{Z}, \quad k \rightarrow \infty .
$$

By assumption of this lemma, the process $\bar{Z}$ behaves as $Z$ until it hits the level $C$. Therefore,

$$
\mathbf{P}\left(\max _{0 \leq t \leq T} \bar{Z}(t)<C\right)=\mathbf{P}\left(\max _{0 \leq t \leq T} Z(t)<C\right) \geq 1-\eta .
$$

The set $\mathcal{A}:=\left\{h \in C[0, T] \mid \max _{t \in[0, T]} h(t)<C\right\}$ is open in $C[0, T]$. For every measurable subset $\mathcal{B} \subseteq \mathcal{A}$, we have:

$$
\mathbf{P}(\bar{Z} \in \mathcal{B})=\mathbf{P}(Z \in \mathcal{B})
$$

Note that we can write

$$
\{Z \in \mathcal{A}\}=\left\{\max _{0 \leq t \leq T} Z(t)<C\right\}=\left\{\max _{0 \leq t \leq T} Z^{C}(t)<C\right\}=\left\{Z^{C} \in \mathcal{A}\right\} .
$$

Therefore, $\mathbf{P}(\bar{Z} \in \mathcal{A})=\mathbf{P}(Z \in \mathcal{A})>1-\eta$. For any open set $\mathcal{G} \subseteq C[0, T]$, we have:

$$
\mathbf{P}\left(X_{n} \in \mathcal{G}\right) \geq \mathbf{P}\left(X_{n} \in \mathcal{A} \cap \mathcal{G}\right)=\mathbf{P}\left(X_{n}^{C} \in \mathcal{A} \cap \mathcal{G}\right) \text {. }
$$

The set $\mathcal{A} \cap \mathcal{G}$ is also open in $C[0, T]$. Applying the portmanteau theorem, see [2, Section 1.2],

$$
\varliminf_{k \rightarrow \infty} \mathbf{P}\left(X_{n_{k}^{\prime}} \in \mathcal{G}\right) \geq \varliminf_{k \rightarrow \infty} \mathbf{P}\left(X_{n_{k}^{\prime}}^{C} \in \mathcal{A} \cap \mathcal{G}\right) \geq \mathbf{P}(\bar{Z} \in \mathcal{A} \cap \mathcal{G}) .
$$

Applying (10) to $\mathcal{B}:=\mathcal{A} \cap \mathcal{G}$, we get:

$$
\mathbf{P}(\bar{Z} \in \mathcal{A} \cap \mathcal{G})=\mathbf{P}(Z \in \mathcal{A} \cap \mathcal{G}) .
$$

Finally, it is straightforward to get the following estimate:

$$
\mathbf{P}(Z \in \mathcal{A} \cap \mathcal{G}) \geq \mathbf{P}(Z \in \mathcal{G})-\mathbf{P}(Z \notin \mathcal{A}) \geq \mathbf{P}(Z \in \mathcal{G})-\eta .
$$


Combining (11), (12), (13), we get:

$$
\varliminf_{k \rightarrow \infty} \mathbf{P}\left(X_{n_{k}^{\prime}} \in \mathcal{G}\right) \geq \mathbf{P}(Z \in \mathcal{G})-\eta .
$$

Now, a small difficulty arises: the subsequence $\left(n_{k}^{\prime}\right)_{k \geq 1}$ might depend on $\eta$. But we can bypass this by taking a sequence $\eta_{m}:=1 / m$ and constructing a sequence of inserted subsequences $\left(n_{k}^{(m)}\right)_{k \geq 1}$ such that $\left(n_{k}^{(m+1)}\right)_{k \geq 1}$ is a subsequence of $\left(n_{k}^{(m)}\right)_{k \geq 1}$. Then

$$
\varliminf_{k \rightarrow \infty} \mathbf{P}\left(X_{n_{k}^{(m)}} \in \mathcal{G}\right) \geq \mathbf{P}(Z \in \mathcal{G})-\frac{1}{m} .
$$

Take the diagonal subsequence $\bar{n}_{k}:=n_{k}^{(k)}, k=1,2, \ldots$ Then

$$
\varliminf_{k \rightarrow \infty} \mathbf{P}\left(X_{\bar{n}_{k}} \in \mathcal{G}\right) \geq \mathbf{P}(Z \in \mathcal{G}) .
$$

This is true for every open set $\mathcal{G} \subseteq C[0, T]$. By another application of the portmanteau theorem, $X_{\bar{n}_{k}} \Rightarrow Z$. We have shown that every increasing sequence $\left(n_{k}\right)_{k \geq 1}$ of positive integers contains a subsequence $\left(\bar{n}_{k}\right)_{k \geq 1}$ such that $X_{\bar{n}_{k}} \Rightarrow Z$. This proves that $X_{n} \Rightarrow Z$.

3.3. Proof of Lemma 3.2. We split this subsection into a few parts. In the first part, we give an overview of the proof: we formulate a series of auxillary lemmata and explain how they fit together to make a proof of Lemma 3.2. In the subsequent parts, we prove each of these auxillary lemmata.

3.3.1. Overview of the proof of Lemma 3.2. The key tool of the proof is convergence of scale functions. The scale function $s_{n}$ for the diffusion $X_{n}$ is defined as follows:

$$
s_{n}(x)=\int_{c_{n}}^{x} \exp \left(-2 \int_{b_{n}}^{y} \frac{f_{n}(z)}{\sigma_{n}^{2}(z)} \mathrm{d} z\right) \mathrm{d} y .
$$

Here, the lower limits $c_{n}$ and $b_{n}$ of integration can be chosen arbitrarily, because the function $s_{n}$ is defined up to an additive and a multiplicative constant. For our purposes, we choose $c_{n}=1$ and $b_{n}>0$ to be determined from the following lemma.

Lemma 3.6. There exists a sequence $\left(b_{n}\right)_{n \geq 1}$ such that $b_{n}>0$ and $b_{n} \rightarrow 0$ as $n \rightarrow \infty$, and for every $x>0$ we have:

$$
\lim _{n \rightarrow \infty} \int_{b_{n}}^{x}\left|\frac{f_{n}(z)}{\sigma^{2}(z)}-\frac{g(z)}{\sigma^{2}(z)}\right| \mathrm{d} z=0 .
$$

Define the limiting scale function:

$$
s(x)=\int_{0}^{x} \exp \left(-2 \int_{0}^{y} \frac{g(z)}{\sigma^{2}(z)} \mathrm{d} z\right) \mathrm{d} y .
$$

Recall that we extended the functions $g$ and $\sigma$ to the real line. Therefore, we can define $s(x)$ using this formula for all $x \in \mathbb{R}$. With this choice of $b_{n}$ as in Lemma 3.6, we can prove convergence of scale functions:

Lemma 3.7. For every $\left[x_{1}, x_{2}\right] \subseteq(0, \infty)$, uniformly on $\left[x_{1}, x_{2}\right]$,

$$
\lim _{n \rightarrow \infty} s_{n}=s, \quad \lim _{n \rightarrow \infty} s_{n}^{\prime}=s^{\prime} .
$$

In addition, for $x<0, \lim _{n \rightarrow \infty} s_{n}(x)=-\infty$.

The next lemma shows that for large $n$, the process $X_{n}$ is more and more likely to hit any negative level only after hitting any fixed level $a>z_{0}$. 
Lemma 3.8. For every $\varepsilon>0$ and $a>z_{0}$, we have:

$$
\lim _{n \rightarrow \infty} \mathbf{P}\left(T_{-\varepsilon}^{(n)}>T_{a}^{(n)}\right)=1
$$

But if we let $a=C$, then

$$
\left\{T_{-\varepsilon}^{(n)}>T_{a}^{(n)}\right\}=\left\{\min _{t \geq 0} X_{n}^{C}(t)>-\varepsilon\right\} \subseteq\left\{\min _{0 \leq t \leq T} X_{n}^{C}(t)>-\varepsilon\right\} .
$$

This completes the proof of Lemma 3.2 .

Remark 3. For every $x>0, \sigma_{n} \rightarrow \sigma$ uniformly on $[0, x]$, and $\sigma$ is a positive continuous function on $[0, x]$. Therefore, there exist $n_{x}, \bar{\sigma}_{x}$ and $\underline{\sigma}_{x}$ such that for $n \geq n_{x}$ and $y \in[0, x]$, we have:

$$
0<\underline{\sigma}_{x} \leq \sigma_{n}(y) \leq \bar{\sigma}_{x}<\infty, \quad 0<\underline{\sigma}_{x} \leq \sigma(y) \leq \bar{\sigma}_{x}<\infty .
$$

3.3.2. Proof of Lemma 3.6. Let us split the proof into the two following lemmata. First, we show convergence of integrals over any fixed subinterval.

Lemma 3.9. For every $\left[x_{1}, x_{2}\right] \subseteq(0, \infty)$,

$$
\lim _{n \rightarrow \infty} \int_{x_{1}}^{x_{2}}\left|\frac{f_{n}(z)}{\sigma_{n}^{2}(z)}-\frac{g(z)}{\sigma^{2}(z)}\right| \mathrm{d} z=0
$$

Then we show the existence of the sequence $\left(b_{n}\right)_{n \geq 1}$ with the required properties. It suffices to prove the following auxillary lemma and apply the results to

$$
g_{n}(z):=\frac{f_{n}(z)}{\sigma_{n}^{2}(z)}-\frac{g(z)}{\sigma^{2}(z)} .
$$

Lemma 3.10. Take a sequence $\left(g_{n}\right)_{n \geq 1}$ of measurable functions $g_{n}:(0, \infty) \rightarrow \mathbb{R}$ such that for every $\left[x_{1}, x_{2}\right] \subseteq(0, \infty)$, we have:

$$
\lim _{n \rightarrow \infty} \int_{x_{1}}^{x_{2}}\left|g_{n}(z)\right| \mathrm{d} z=0
$$

Then there exists a sequence $\left(b_{n}\right)_{n \geq 1}$ of positive numbers with $b_{n} \rightarrow 0$ and for all $x_{0}>0$,

$$
\lim _{n \rightarrow \infty} \int_{b_{n}}^{x_{0}}\left|g_{n}(z)\right| \mathrm{d} z=0
$$

Proof of Lemma 3.9. We have:

$$
\begin{aligned}
\int_{x_{1}}^{x_{2}}\left|\frac{f_{n}(z)}{\sigma_{n}^{2}(z)}-\frac{g(z)}{\sigma^{2}(z)}\right| \mathrm{d} z & \leq \int_{x_{1}}^{x_{2}} \frac{\left|f_{n}(z)-g(z)\right|}{\sigma_{n}^{2}(z)} \mathrm{d} z \\
& +\int_{x_{1}}^{x_{2}}|g(z)|\left|\sigma_{n}^{-2}(z)-\sigma^{-2}(z)\right| \mathrm{d} z:=I_{1}(n)+I_{2}(n) .
\end{aligned}
$$

Recall from (14) that $\sigma_{n}^{2}(y) \geq \underline{\sigma}_{x_{2}}^{2}$ for all $y \in\left[x_{1}, x_{2}\right]$ and $n \geq n_{x_{2}}$. Using the condition (ii) of Theorem 2.1, we get: as $n \rightarrow \infty$,

$$
I_{1}(n) \leq \underline{\sigma}_{x_{2}}^{-2} \int_{x_{1}}^{x_{2}}\left|f_{n}(z)-g(z)\right| \mathrm{d} z \rightarrow 0 .
$$

Since $\sigma_{n}^{2} \rightarrow \sigma^{2}$ uniformly on $\left[x_{1}, x_{2}\right]$, by Lemma 4.1 (ii), $\sigma_{n}^{-2} \rightarrow \sigma^{-2}$ uniformly on $\left[x_{1}, x_{2}\right]$. And the function $g$ is continuous (therefore, it is bounded) on $\left[x_{1}, x_{2}\right]$. Thus, uniformly on $\left[x_{1}, x_{2}\right]$,

$$
|g(z)|\left|\sigma_{n}^{-2}(z)-\sigma^{-2}(z)\right| \rightarrow 0 .
$$

Which proves that $I_{2}(n) \rightarrow 0$ as $n \rightarrow \infty$. 
Proof of Lemma 3.10. For $b \in(0,1)$ and $n=1,2, \ldots$, let

$$
M(b, n):=\int_{b}^{1}\left|g_{n}(z)\right| \mathrm{d} z .
$$

Then for every $b \in(0,1)$, we have: $\lim _{n \rightarrow \infty} M(b, n)=0$. Take $b:=1 / k$ and find $n_{k}$ such that for $n \geq n_{k}$ we have: $M\left(1 / k, n_{k}\right) \leq 1 / k$. Now, let $\bar{n}_{1}:=n_{1}$ and $\bar{n}_{k+1}:=\max \left(\bar{n}_{1}, \ldots, \bar{n}_{k}, n_{k+1}\right)+1$. Then $\bar{n}_{1}<\bar{n}_{2}<\ldots$, and for $n \geq \bar{n}_{k}, M\left(1 / k, \bar{n}_{k}\right) \leq 1 / k$. Define the sequence $\left(b_{n}\right)_{n \geq 1}$ as follows: $b_{n}=k^{-1}$ for $\bar{n}_{k} \leq n<\bar{n}_{k+1}$. For $n<\bar{n}_{1}$, just let $b_{n}=1$. Then we get: for $\bar{n}_{k} \leq n<\bar{n}_{k+1}$,

$$
M\left(b_{n}, n\right)=M(1 / k, n) \leq \frac{1}{k}
$$

Therefore, $M\left(b_{n}, n\right) \rightarrow 0$ as $n \rightarrow \infty$. This proves the statement of the lemma for $x_{0} \leq 1$. For $y>1$, just note that

$$
\int_{1}^{x_{0}}\left|g_{n}(z)\right| \mathrm{d} z \rightarrow 0
$$

Thus, we can represent the original integral as

$$
\int_{b_{n}}^{x_{0}}\left|g_{n}(z)\right| \mathrm{d} z=\int_{b_{n}}^{1}\left|g_{n}(z)\right| \mathrm{d} z+\int_{1}^{x_{0}}\left|g_{n}(z)\right| \mathrm{d} z \rightarrow 0 .
$$

3.3.3. Proof of Lemma 3.7. First, let us show that for $x>0, \lim _{n \rightarrow \infty} s_{n}^{\prime}(x)=s^{\prime}(x)$. Indeed, by Lemma 3.6, we get: as $n \rightarrow \infty$,

$$
\int_{b_{n}}^{x}\left|\frac{f_{n}(z)}{\sigma_{n}^{2}(z)}-\frac{g(z)}{\sigma^{2}(z)}\right| \mathrm{d} z \rightarrow 0
$$

But we have:

$$
\left|\int_{b_{n}}^{x} \frac{f_{n}(z)}{\sigma_{n}^{2}(z)} \mathrm{d} z-\int_{0}^{x} \frac{g(z)}{\sigma^{2}(z)} \mathrm{d} z\right| \leq \int_{b_{n}}^{x}\left|\frac{f_{n}(z)}{\sigma_{n}^{2}(z)}-\frac{g(z)}{\sigma^{2}(z)}\right| \mathrm{d} z+\int_{0}^{b_{n}} \frac{|g(z)|}{\sigma^{2}(z)} \mathrm{d} z .
$$

We need only to show that, as $n \rightarrow \infty$,

$$
\int_{0}^{b_{n}} \frac{|g(z)|}{\sigma^{2}(z)} \mathrm{d} z \rightarrow 0
$$

To prove (17), we need only to note that $b_{n} \rightarrow 0$ as $n \rightarrow \infty, \sigma^{-2}(z) \leq \underline{\sigma}_{1}^{-2}$ for $z \in[0,1]$ because of (14), and the function $g$ is continuous. From (16), it follows that, as $n \rightarrow \infty$, for all $x>0$,

$$
\log s_{n}^{\prime}(x)=-2 \int_{b_{n}}^{x} \frac{f_{n}(z)}{\sigma_{n}^{2}(z)} \mathrm{d} z \rightarrow-2 \int_{0}^{x} \frac{g(z)}{\sigma^{2}(z)} \mathrm{d} z=\log s^{\prime}(x) .
$$

Therefore, $s_{n}^{\prime}(x) \rightarrow s^{\prime}(x)$. Now, we show this convergence is uniform on every $\left[x_{1}, x_{2}\right] \subseteq(0, \infty)$. There exists an $n_{0}$ such that for $n \geq n_{0}$ we have: $b_{n}<x_{1}$. Then for $x \in\left[x_{1}, x_{2}\right]$, from (16)), we get:

$$
\left|\log s_{n}^{\prime}(x)-\log s^{\prime}(x)\right| \leq \int_{b_{n}}^{x_{2}}\left|\frac{f_{n}(z)}{\sigma_{n}^{2}(z)}-\frac{g(z)}{\sigma^{2}(z)}\right| \mathrm{d} z+\int_{0}^{b_{n}} \frac{|g(z)|}{\sigma^{2}(z)} \mathrm{d} z \rightarrow 0, \quad n \rightarrow \infty .
$$

Therefore, $\log s_{n}^{\prime}(x) \rightarrow \log s^{\prime}(x)$ uniformly on $\left[x_{1}, x_{2}\right]$. The function $\log s^{\prime}(x)$ is continuous on $\left[x_{1}, x_{2}\right]$, and therefore is bounded on this segment. There exist $n_{2}$ and $C_{0}>0$ such that for $x \in\left[x_{1}, x_{2}\right], n \geq n_{2}$, we have:

$$
\left|\log s_{n}^{\prime}(x)\right| \leq C_{0}, \quad\left|\log s^{\prime}(x)\right| \leq C_{0} .
$$

The function $z \mapsto e^{z}$ is uniformly continuous on $\left[-C_{0}, C_{0}\right]$, and $\log s_{n}^{\prime}(x) \rightarrow \log s_{n}(x)$ uniformly on $\left[x_{1}, x_{2}\right]$. Therefore, uniformly on $\left[x_{1}, x_{2}\right]$,

$$
s_{n}^{\prime}(x)=e^{\log s_{n}^{\prime}(x)} \rightarrow e^{\log s^{\prime}(x)}=s^{\prime}(x), \quad n \rightarrow \infty, \quad \text { and }
$$




$$
s_{n}(x)=\int_{1}^{x} s_{n}^{\prime}(z) \mathrm{d} z \rightarrow s(x)=\int_{1}^{x} s^{\prime}(z) \mathrm{d} z, \quad n \rightarrow \infty,
$$

and the convergence is uniform on every $\left[x_{1}, x_{2}\right] \subseteq(0, \infty)$. Finally, let us show that $s_{n}(x) \rightarrow-\infty$ for $x<0$. Indeed, if we prove that $s_{n}^{\prime}(x) \rightarrow \infty$ for $x<0$, then $s_{n}^{\prime}(x) \geq 0$, and by Fatou's lemma,

$$
-s_{n}(x)=-\int_{1}^{x} s_{n}^{\prime}(y) \mathrm{d} y=\int_{x}^{1} s_{n}^{\prime}(y) \mathrm{d} y \geq \int_{x}^{0} s_{n}^{\prime}(y) \mathrm{d} y \rightarrow \infty .
$$

It suffices to show that

$$
\log s_{n}^{\prime}(x)=-2 \int_{b_{n}}^{x} \frac{f_{n}(z)}{\sigma_{n}^{2}(z)} \mathrm{d} z=2 \int_{x}^{b_{n}} \frac{f_{n}(z)}{\sigma_{n}^{2}(z)} \mathrm{d} z \rightarrow \infty .
$$

But we have:

$$
\int_{x}^{b_{n}} \frac{f_{n}(z)}{\sigma_{n}^{2}(z)} \mathrm{d} z=\left(\int_{x}^{1}-\int_{b_{n}}^{1}\right) \frac{f_{n}(z)}{\sigma_{n}^{2}(z)} \mathrm{d} z, \text { and } \int_{x}^{1} \frac{f_{n}(z)}{\sigma_{n}^{2}(z)} \mathrm{d} z \rightarrow \infty .
$$

From the relation (16) applied to $x=1$, we get:

$$
\int_{b_{n}}^{1} \frac{f_{n}(z)}{\sigma_{n}^{2}(z)} \mathrm{d} z \rightarrow \int_{0}^{1} \frac{g(z)}{\sigma^{2}(z)} \mathrm{d} z
$$

Combining (19) and (20), we get (18).

3.3.4. Proof of Lemma 3.8. The process $s_{n}\left(X_{n}(\cdot)\right)$ is a local martingale. Therefore, the process $s_{n}\left(X_{n}\left(\cdot \wedge T_{-\varepsilon}^{(n)} \wedge T_{a}^{(n)}\right)\right)$ is a bounded martingale. By the optional stopping theorem,

$$
\mathbf{P}\left(T_{-\varepsilon}^{(n)}<T_{a}^{(n)}\right)=\frac{s_{n}(a)-s_{n}\left(z_{0}\right)}{s_{n}(a)-s_{n}(-\varepsilon)}
$$

But by Lemma 3.7, $s_{n}(a) \rightarrow s(a), s_{n}\left(z_{0}\right) \rightarrow s\left(z_{0}\right), s_{n}(-\varepsilon) \rightarrow-\infty$. Therefore, the quantity in (21) converges to zero, which completes the proof.

3.4. Proof of Lemma 3.3. Since $X_{n}^{C}(0)=z_{n} \rightarrow z_{0}=Z(0)$, by the Arzela-Ascoli criterion it suffices to show that for all $\varepsilon>0$,

$$
\lim _{\delta \rightarrow 0} \sup _{n \geq 1} \mathbf{P}\left(\omega\left(X_{n}^{C},[0, T], \delta\right)>3 \varepsilon\right)=0 .
$$

Note that $X_{n}^{C}$ is constant on $\left[T_{C}^{(n)}, T\right]$, and is equal to $X_{n}$ on $\left[0, T_{C}^{(n)}\right]$. Therefore, $\omega\left(X_{n}^{C},[0, T], \delta\right)=$ $\omega\left(X_{n},\left[0, T_{C}^{(n)}\right], \delta\right)$. Assume $\omega\left(X_{n},\left[0, T_{C}^{(n)}\right], \delta\right)>3 \varepsilon$, and $\min _{[0, T]} X_{n}^{C}>-\varepsilon$. Then there exist $t_{1}, t_{2} \in\left[0, T_{C}^{(n)}\right]$ such that $\left|t_{1}-t_{2}\right| \leq \delta$, and $\left|X_{n}\left(t_{1}\right)-X_{n}\left(t_{2}\right)\right|>3 \varepsilon$. Assume without loss of generality $X_{n}\left(t_{1}\right)-X_{n}\left(t_{2}\right)>3 \varepsilon$. Since $X_{n}\left(t_{2}\right)>-\varepsilon$, we get: $X_{n}\left(t_{1}\right)>2 \varepsilon$. By continuity of $X_{n}$, we can find $t_{3}$ between $t_{1}$ and $t_{2}$ such that $X_{n}\left(t_{3}\right)=X_{n}\left(t_{2}\right)+2 \varepsilon>3 \varepsilon-\varepsilon=\varepsilon$. And we can find $t_{4}$ between $t_{1}$ and $t_{3}$ such that $X_{n}\left(t_{4}\right)=X_{n}\left(t_{3}\right)+\varepsilon$. Then

$$
X_{n}\left(t_{3}\right), X_{n}\left(t_{4}\right) \in[\varepsilon, C], \quad\left|t_{4}-t_{3}\right| \leq\left|t_{1}-t_{2}\right| \leq \delta
$$

But $s_{n}^{\prime}(x)>0$ for $x \in[\varepsilon, C]$ and $n=1,2, \ldots$, and $s_{n}^{\prime}(x) \rightarrow s^{\prime}(x)>0$ uniformly on $[\varepsilon, C]$. Therefore, there exists $C_{1}>0$ such that for all $n=1,2, \ldots, s_{n}^{\prime}(x) \geq C_{1}, x \in[\varepsilon, C]$. We have:

$$
s_{n}\left(X_{n}\left(t_{4}\right)\right)-s_{n}\left(X_{n}\left(t_{3}\right)\right) \geq C_{1}\left(X_{n}\left(t_{4}\right)-X_{n}\left(t_{3}\right)\right) \geq C_{1} \varepsilon
$$

But the process $s_{n}\left(X_{n}(\cdot)\right)$ satisfies the following stochastic equation:

$$
\mathrm{d} s_{n}\left(X_{n}(t)\right)=s_{n}^{\prime}\left(X_{n}(t)\right) \sigma_{n}\left(X_{n}(t)\right) \mathrm{d} W(t),
$$

with the coefficient satisfying

$$
\left|s_{n}^{\prime}(x) \sigma_{n}(x)\right| \leq C_{0}, \quad x \in[\varepsilon, C], \quad n=1,2, \ldots
$$


By Lemma 4.3 (proved in the Appendix), we have: for a certain real constant $\varkappa>0$,

$$
\mathbf{P}\left(\exists t_{3}, t_{4} \in[0, T]:\left|t_{3}-t_{4}\right| \leq \delta, s_{n}\left(X_{n}\left(t_{4}\right)\right)-s_{n}\left(X_{n}\left(t_{3}\right)\right)=C_{1} \varepsilon\right) \leq \varkappa \frac{C_{0}^{4} T}{\left(C_{1} \varepsilon\right)^{2}} \delta .
$$

In other words, because of the estimate (24), the probability of the event (22) is very small for small $\delta$. Therefore, for all $n \geq 1$,

$$
\mathbf{P}\left(\omega\left(X_{n}^{C},[0, T], \delta\right) \geq 3 \varepsilon\right) \leq \varkappa \frac{C_{0}^{4} T}{\left(C_{1} \varepsilon\right)^{2}} \delta .
$$

Letting $\delta \rightarrow 0$, we complete the proof of Lemma 3.3.

3.5. Proof of Lemma 3.4. For $t \in[0, T]$ and $n=1,2, \ldots$, let

$$
M_{n}(t):=\int_{0}^{t \wedge T_{C}^{(n)}} \sigma_{n}\left(X_{n}(u)\right) \mathrm{d} W(u), \quad M(t):=\int_{0}^{t \wedge T_{C}^{\bar{Z}}} \sigma(\bar{Z}(u)) \mathrm{d} W(u) .
$$

These are continuous local martingales. We can express them as time-changed Brownian motions:

$$
M_{n}(t)=B_{n}\left(\left\langle M_{n}\right\rangle_{t}\right), \quad M(t)=B\left(\langle M\rangle_{t}\right),
$$

where $B_{n}, B$ are standard Brownian motions, and the quadratic variations $\left\langle M_{n}\right\rangle_{t}$ and $\langle M\rangle_{t}$ of $M_{n}$ and $M$ respectively can be expressed explicitly as

$$
\left\langle M_{n}\right\rangle_{t}=\int_{0}^{t \wedge T_{C}^{(n)}} \sigma_{n}^{2}\left(X_{n}(u)\right) \mathrm{d} u, \quad\langle M\rangle_{t}=\int_{0}^{t \wedge T_{C}^{\bar{Z}}} \sigma^{2}(\bar{Z}(u)) \mathrm{d} u .
$$

Note that $\sigma^{2}(\bar{Z}(u)) \leq \bar{\sigma}_{C}^{2}$ for $u \in\left[0, T_{C}^{\bar{Z}}\right]$ (see Remark[3). Therefore, $\langle M\rangle_{t} \leq \bar{\sigma}_{C}^{2} T$ for $t \in[0, T], n=$ $1,2, \ldots$ We can conclude that the sequence $\left(X_{n_{k}}, B_{n_{k}}\right)_{k \geq 1}$ is tight in $C([0, T], \mathbb{R}) \times C\left(\left[0, \bar{\sigma}_{C}^{2} T+1\right], \mathbb{R}\right)$. It has a weak limit point for some subsequence $\left(n_{k}^{\prime}\right)$ of $\left(n_{k}\right)$ :

$$
\left(X_{n_{k}^{\prime}}, B_{n_{k}^{\prime}}\right) \Rightarrow(\bar{Z}, \bar{B}) \text {. }
$$

The process $\bar{B}$ is a weak limit of a sequence of standard Brownian motions, and therefore is itself a standard Brownian motion. By the Skorohod representation theorem, we can switch from weak to pathwise convergence (a.s.) after changing the probability space. Since $X_{n_{k}^{\prime}} \rightarrow \bar{Z}$, then by Lemma 3.2 we have: $\bar{Z}(t) \geq 0$ for all $t \in[0, T]$.

Lemma 3.11. The hitting times of the level $C$ satisfy the following inequality a.s.:

$$
T_{C}^{\bar{Z}} \leq \varliminf_{k \rightarrow \infty} T_{C}^{\left(n_{k}^{\prime}\right)}
$$

Proof. Indeed, assume the converse. Then there exists a subsequence $\left(\bar{n}_{k}\right)_{k \geq 1}$ of $\left(n_{k}^{\prime}\right)_{k \geq 1}$ and a number $t_{0} \in\left[0, T_{C}^{\bar{Z}}\right)$ such that $T_{C}^{\left(\bar{n}_{k}\right)} \leq t_{0}$. But this means that $T_{C}^{\left(\bar{n}_{k}\right)} \leq t_{0}<T$, and

$$
X_{\bar{n}_{k}}^{C}\left(t_{0}\right)=X_{\bar{n}_{k}}^{C}\left(T_{C}^{\left(\bar{n}_{k}\right)}\right)=C \text {. }
$$

Letting $k \rightarrow \infty$, we get: $\bar{Z}\left(t_{0}\right)=C$. But this contradicts the fact that $T_{C}^{\bar{Z}}>t_{0}$. This completes the proof of this lemma.

By Lemma 4.2, $\sigma_{n_{k}^{\prime}}\left(X_{n_{k}^{\prime}}(t)\right) \rightarrow \sigma(\bar{Z}(t))$ a.s. uniformly on $[0, T]$. Hence a.s. uniformly on $[0, T]$,

$$
\left\langle M_{n_{k}^{\prime}}\right\rangle_{t}=\int_{0}^{t} \sigma_{n_{k}^{\prime}}^{2}\left(X_{n_{k}^{\prime}}(u)\right) \mathrm{d} u \rightarrow\langle M\rangle_{t}=\int_{0}^{t} \sigma^{2}(\bar{Z}(u)) \mathrm{d} u .
$$


Applying Lemma 4.1(iv), we get:

$$
M_{n_{k}^{\prime}}(t)=B_{n_{k}^{\prime}}\left(\left\langle M_{n_{k}^{\prime}}\right\rangle_{t}\right) \rightarrow M(t)=B\left(\langle M\rangle_{t}\right)
$$

a.s. uniformly on $[0, T]$. Also, $g$ is continuous on $\mathbb{R}$, and $X_{n_{k}^{\prime}} \rightarrow \bar{Z}$ uniformly on $[0, T]$, hence $g\left(X_{n_{k}^{\prime}}(t)\right) \rightarrow g(\bar{Z}(t))$ uniformly on $[0, T]$. Therefore,

$$
\int_{0}^{t} g\left(X_{n_{k}^{\prime}}(u)\right) \mathrm{d} u \rightarrow \int_{0}^{t} g(\bar{Z}(u)) \mathrm{d} u, n \rightarrow \infty,
$$

uniformly on $[0, T]$. Finally, $z_{n} \rightarrow z$. Combining this with (25) and (26), we get: $Z_{n_{k}^{\prime}} \rightarrow Z$ uniformly on $[0, T]$. Finally, weak convergence in $C[0, T]$ follows from uniform convergence.

3.6. Proof of Lemma 3.5. This proof is a bit lengthy, so we organize it in the same way as the proof of Theorem 2.1? In the first subsection, we split the proof into a few lemmata, and in the following subsections, we prove each of these lemmata.

3.6.1. Outline of the proof of Lemma 3.5. Fix a subinterval $\left[t_{1}, t_{2}\right] \subseteq[0, T]$ and a number $\eta>0$.

Lemma 3.12. Suppose we have already shown that

$$
\mathbf{P}\left(\left|\bar{L}\left(t_{2}\right)-\bar{L}\left(t_{1}\right)\right|>\eta, \min _{t \in\left[t_{1}, t_{2}\right]} \bar{Z}(t)>\eta\right)=0 .
$$

Then the statement of Lemma 3.5 is true.

Consider the subset

$$
\mathcal{G}:=\left\{(x, y) \in C[0, T] \times C[0, T] \mid y\left(t_{2}\right)>y\left(t_{1}\right)+\eta, \min _{\left[t_{1}, t_{2}\right]} x(t)>\eta\right\} .
$$

It is open in $C[0, T] \times C[0, T]$. Suppose we proved that

$$
\lim _{k \rightarrow \infty} \mathbf{P}\left(\left(X_{n_{k}^{\prime}}^{C}, L_{n_{k}^{\prime}}\right) \in \mathcal{G}\right)=0 .
$$

Then by the portmanteau theorem, $\mathbf{P}((\bar{Z}, \bar{L}) \in \mathcal{G})=0$, which is equivalent to (27). Therefore, it suffices to show (28) to prove (27). Define $s^{-1}$ to be the inverse function of $s$, with the domain $(s(-\infty), s(\infty))$. Let

$$
\varepsilon_{n}:=\max _{x \in[\eta, C]}\left|s^{-1}\left(s_{n}(x)\right)-x\right| .
$$

Then $\varepsilon_{n} \rightarrow 0$ : indeed, $s_{n} \rightarrow s$ uniformly on $[\eta, C]$, and by Lemma 4.1 we get: $s^{-1}\left(s_{n}(x)\right) \rightarrow$ $s^{-1}(s(x))=x$ uniformly on $[\eta, C]$. Define also

$$
Y_{n}(t) \equiv s^{-1}\left(s_{n}\left(X_{n}^{C}(t)\right)\right)-Z_{n}\left(t \wedge T_{C}^{(n)}\right) .
$$

The next two lemmata form the crux of the proof of Lemma 3.5.

Lemma 3.13. For large enough $n$, the process $Y_{n}$ is well defined, and for $t<T_{C}^{(n)}$, we have:

$$
\mathrm{d} Y_{n}(t)=\beta_{n}\left(X_{n}^{C}(t)\right) \mathrm{d} t+\alpha_{n}\left(X_{n}^{C}(t)\right) \mathrm{d} W(t),
$$

where the drift and diffusion coefficients $\beta_{n}(\cdot), \alpha_{n}(\cdot)$ are given by

$$
\begin{gathered}
\alpha_{n}(x):=\frac{s_{n}^{\prime}(x) \sigma_{n}(x)}{s^{\prime}\left(s^{-1}\left(s_{n}(x)\right)\right)}-\sigma_{n}(x), \\
\beta_{n}(x)=\frac{s_{n}^{\prime 2}(x) \sigma_{n}^{2}(x)}{\sigma^{2}\left(s^{-1}\left(s_{n}(x)\right)\right) s^{\prime 2}\left(s^{-1}\left(s_{n}(x)\right)\right)} g\left(s^{-1}\left(s_{n}(x)\right)\right)-g(x) .
\end{gathered}
$$

Lemma 3.14. For every interval $\left[x_{1}, x_{2}\right] \subseteq(0, \infty)$, we have: $\alpha_{n}, \beta_{n} \rightarrow 0$ uniformly on $\left[x_{1}, x_{2}\right]$. 
From (30) and the definition (9) of $Z_{n}$, we get:

$$
Y_{n}(t) \equiv s^{-1}\left(s_{n}\left(X_{n}^{C}(t)\right)\right)-X_{n}^{C}(t)+L_{n}(t) .
$$

From the definition (29) of $\varepsilon_{n}$, we get:

$$
\left\{\min _{\left[t_{1}, t_{2}\right]} X_{n}^{C}(t)>\eta\right\} \subseteq\left\{\max _{t_{1} \leq t \leq t_{2}}\left|s^{-1}\left(s_{n}\left(X_{n}^{C}(t)\right)\right)-X_{n}^{C}(t)\right| \leq \varepsilon_{n}\right\} .
$$

Combining (32) and (33), we have:

$$
\left\{\min _{\left[t_{1}, t_{2}\right]} X_{n}^{C}(t)>\eta\right\} \subseteq\left\{\max _{t_{1} \leq t \leq t_{2}}\left|Y_{n}(t)-L_{n}(t)\right| \leq \varepsilon_{n}\right\} .
$$

Lemma 3.15. Fix $\varepsilon>0$. We introduce the following event:

$$
A_{n}(\varepsilon):=\left\{\exists t_{1}^{\prime}, t_{2}^{\prime} \in\left[t_{1}, t_{2}\right]|| Y_{n}\left(t_{2}^{\prime}\right)-Y_{n}\left(t_{1}^{\prime}\right) \mid \geq \varepsilon, \min _{\left[t_{1}, t_{2}\right]} X_{n}^{C}>\eta\right\} .
$$

Then $\mathbf{P}\left(A_{n}(\varepsilon)\right) \rightarrow 0$ as $n \rightarrow \infty$.

Now, we can complete the proof of (28). Assume the following event has happened:

$$
A_{n}:=\left\{\left|L_{n}\left(t_{2}\right)-L_{n}\left(t_{1}\right)\right|>\eta, \min _{\left[t_{1}, t_{2}\right]} X_{n}^{C}>\eta\right\} .
$$

Since $\varepsilon_{n} \rightarrow 0$, there exists $n_{1}$ such that for $n \geq n_{1}$, we have: $\varepsilon_{n}<\eta / 3$. From (34), we get that, for $n \geq n_{1}$, the following event also has happened:

$$
A_{n}^{\prime}:=\left\{\left|Y_{n}\left(t_{2}\right)-Y_{n}\left(t_{1}\right)\right|>\eta-2 \varepsilon_{n}>\eta / 3, \min _{\left[t_{1}, t_{2}\right]} X_{n}^{C}>\eta\right\} .
$$

Apply Lemma 3.15 for $\varepsilon=\eta / 3$ and get that $\mathbf{P}\left(A_{n}^{\prime}\right) \rightarrow 0$. Therefore, $\mathbf{P}\left(A_{n}\right) \rightarrow 0$, which completes the proof of (28), as well as the proof of Lemma 3.5.

3.6.2. Proof of Lemma 3.12. First, let us show $\bar{L}$ cannot increase when $\bar{Z}>0$. Assume the converse; then there exists a subinterval $\left[t_{1}, t_{2}\right] \subseteq[0, T]$ such that $\bar{L}\left(t_{2}\right)>\bar{L}\left(t_{1}\right)$, and $\bar{Z}(t)>0$ for $t \in\left[t_{1}, t_{2}\right]$. Since the function $\bar{L}$ is continuous, there exist rational $q_{1}, q_{2} \in\left(t_{1}, t_{2}\right)$ such that $q_{1}<q_{2}$ and $\bar{L}\left(q_{2}\right)>\bar{L}\left(q_{1}\right)$. Find a rational $q>0$ small enough so that $\bar{L}\left(q_{2}\right)>\bar{L}\left(q_{1}\right)+q$, and $\min _{\left[t_{1}, t_{2}\right]} \bar{Z}>q$. Let

Then

$$
F\left(q_{1}, q_{2}, q\right):=\left\{\bar{L}\left(t_{2}\right)>\bar{L}\left(t_{1}\right)+q, \min _{\left[t_{1}, t_{2}\right]} \bar{Z}>q\right\}
$$

$$
\left\{\int_{0}^{T} \bar{Z}(t) \mathrm{d} \bar{L}(t)>0\right\}=\bigcup F\left(q_{1}, q_{2}, q\right),
$$

where the union is taken over all rational $q, q_{1}, q_{2}$ such that $0 \leq q_{1}<q_{2} \leq T$, and $q>0$. By the assumption of the lemma, $\mathbf{P}\left(F\left(q_{1}, q_{2}, q\right)\right)=0$. Observe that this union is countable to complete the proof.

Next, let us show $\bar{L}$ is a.s. nondecreasing. It suffices to prove that for every $\delta>0$, for all $t_{1}, t_{2} \in[0, T]$ such that $t_{1}<t_{2} \leq T_{C}^{\bar{Z}}$, we have:

$$
\bar{L}\left(t_{2}\right)-\bar{L}\left(t_{1}\right) \geq-\delta\left(t_{2}-t_{1}\right)
$$

Then, in turn, it suffices to show that for every $t_{0} \in\left(0, T_{C}^{\bar{Z}}\right)$, there exists a neighborhood $\left(t_{0}-\right.$ $\left.\varepsilon, t_{0}+\varepsilon\right) \subseteq[0, T]$ such that the property (35) is satisfied in this neighborhood. Indeed, if we proved this, then the standard compactness argument gives us (35) on the whole $[0, T]$. Assume $\bar{Z}\left(t_{0}\right)>0$. By continuity of $\bar{Z}$, there exists a neighborhood of $t_{0}$ in which $\bar{Z}>0$ and therefore, 
by Lemma 3.5, the process $\bar{L}$ is constant. Then it obviously satisfies (35) in this neighborhood. Now, assume $\bar{Z}\left(t_{0}\right)=0$. From condition (iii) of Theorem 2.1, we have: there exist $\delta_{0}>0$ and $n_{0}$ such that for $x \in\left(-\delta_{0}, \delta_{0}\right)$ and $n \geq n_{0}$, we get: $f_{n}(x) \geq g(0)-\delta$. By continuity of $\bar{Z}$, there exists $\varepsilon>0$ such that $\bar{Z}(t)<\delta_{0} / 2$ for $t \in\left(t_{0}-\varepsilon, t_{0}+\varepsilon\right)$. By the uniform convergence $X_{n_{k}^{\prime}}^{C} \rightarrow \bar{Z}$ on $\left(t_{0}-\varepsilon, t_{0}+\varepsilon\right)$, there exists $k_{0}$ such that for $k \geq k_{1}$ we get:

$$
-\delta_{0}<X_{n_{k}^{\prime}}^{C}(t)<\delta_{0}, \quad t \in\left(t_{0}-\varepsilon, t_{0}+\varepsilon\right) .
$$

We can find $k_{2}$ large enough so that for $k \geq k_{2}$, we have: $n_{k}^{\prime} \geq n_{0}$. Then we have: for $k \geq k_{1} \vee k_{2}$,

$$
f_{n_{k}^{\prime}}\left(X_{n_{k}^{\prime}}^{C}(t)\right)-g\left(X_{n_{k}^{\prime}}^{C}(t)\right)>-\delta
$$

and for $t_{1}, t_{2} \in\left(t_{0}-\varepsilon, t_{0}+\varepsilon\right), t_{1}<t_{2}$, we get:

$$
\bar{L}_{n_{k}^{\prime}}\left(t_{2}\right)-\bar{L}_{n_{k}^{\prime}}\left(t_{1}\right)=\int_{t_{1}}^{t_{2}}\left[f_{n_{k}^{\prime}}\left(X_{n_{k}^{\prime}}^{C}(t)\right)-g\left(X_{n_{k}^{\prime}}^{C}(t)\right)\right] \mathrm{d} t \geq-\delta\left(t_{2}-t_{1}\right) .
$$

Letting $k \rightarrow \infty$, we get (35). The proof is complete.

3.6.3. Proof of Lemma 3.13. First, let us show that the process $Y_{n}$ is well defined for sufficiently large $n$ : that is, for every $\left[x_{1}, x_{2}\right] \subseteq(0, \infty)$ there exists $n_{0}$ such that for $n \geq n_{0}$, the domain $(s(-\infty), s(\infty))$ of $s^{-1}$ contains $\left[s_{n}\left(x_{1}\right), s_{n}\left(x_{2}\right)\right]$. Indeed, there exists $\varepsilon>0$ such that $\left[s\left(x_{1}\right)-\right.$ $\left.\varepsilon, s\left(x_{2}\right)+\varepsilon\right] \subseteq(s(-\infty), s(\infty))$, because $s^{\prime}(x)>0$ for all $x \in \mathbb{R}$. Moreover, there exists $n_{0}$ such that for $n \geq n_{0}$ we have:

$$
s_{n}\left(x_{1}\right)>s\left(x_{1}\right)-\varepsilon, \quad s_{n}\left(x_{2}\right)<s\left(x_{2}\right)+\varepsilon .
$$

Therefore, for $n \geq n_{0}$ we have: $\left[s_{n}\left(x_{1}\right), s_{n}\left(x_{2}\right)\right] \subseteq(s(-\infty), s(\infty))$. This proves that $Y_{n}$ is well defined for $n \geq n_{0}$.

Let us show the representation (31) for $\mathrm{d} Y_{n}(t)$. For all $x \in \mathbb{R}$ and $n=1,2, \ldots$, we have:

$$
s_{n}^{\prime}(x)=\exp \left(-2 \int_{b_{n}}^{x} \frac{f_{n}(z)}{\sigma_{n}^{2}(z)} \mathrm{d} z\right), \quad s^{\prime}(x)=\exp \left(-2 \int_{0}^{x} \frac{g(z)}{\sigma^{2}(z)} \mathrm{d} z\right) .
$$

Therefore, for $y \in(s(-\infty), s(\infty))$,

$$
\left(s^{-1}\right)^{\prime}(y)=\frac{1}{s^{\prime}\left(s^{-1}(y)\right)}=\exp \left(2 \int_{0}^{s^{-1}(y)} \frac{g(z)}{\sigma^{2}(z)} \mathrm{d} z\right)
$$

and

$$
\begin{gathered}
\left(s^{-1}\right)^{\prime \prime}(y)=\exp \left(2 \int_{0}^{s^{-1}(y)} \frac{g(z)}{\sigma^{2}(z)} \mathrm{d} z\right) \cdot 2 \frac{g\left(s^{-1}(y)\right)}{\sigma^{2}\left(s^{-1}(y)\right)} \cdot\left(s^{-1}(y)\right)^{\prime} \\
=\exp \left(4 \int_{0}^{s^{-1}(y)} \frac{g(z)}{\sigma^{2}(z)} \mathrm{d} z\right) \cdot 2 \frac{g\left(s^{-1}(y)\right)}{\sigma^{2}\left(s^{-1}(y)\right)} .
\end{gathered}
$$

By Itô's formula, applied to the process $s_{n}\left(X_{n}^{C}(\cdot)\right)$ from (23)

$$
\begin{aligned}
\mathrm{d} s^{-1}\left(s_{n}\left(X_{n}^{C}(t)\right)\right) & =\left(s^{-1}\right)^{\prime}\left(s_{n}\left(X_{n}^{C}(t)\right)\right) s_{n}^{\prime}\left(X_{n}^{C}(t)\right) \sigma_{n}\left(X_{n}^{C}(t)\right) \mathrm{d} W(t) \\
& +\frac{1}{2}\left(s^{-1}\right)^{\prime \prime}\left(s_{n}\left(X_{n}^{C}(t)\right)\right)\left[s_{n}^{\prime}\left(X_{n}^{C}(t)\right) \sigma_{n}\left(X_{n}^{C}(t)\right)\right]^{2} \mathrm{~d} t \\
& =\exp \left(2 \int_{0}^{s^{-1}\left(s_{n}\left(X_{n}^{C}(t)\right)\right)} \frac{g(z)}{\sigma^{2}(z)} \mathrm{d} z-2 \int_{b_{n}}^{X_{n}^{C}(t)} \frac{f_{n}(z)}{\sigma_{n}^{2}(z)} \mathrm{d} z\right) \sigma_{n}\left(X_{n}^{C}(t)\right) \mathrm{d} W(t)
\end{aligned}
$$




$$
\begin{aligned}
& +\frac{1}{2} \exp \left(4 \int_{0}^{s^{-1}\left(s_{n}\left(X_{n}^{C}(t)\right)\right)} \frac{g(z)}{\sigma^{2}(z)} \mathrm{d} z\right) 2 \frac{g\left(s^{-1}\left(s_{n}\left(X_{n}^{C}(t)\right)\right)\right)}{\sigma^{2}\left(s^{-1}\left(s_{n}\left(X_{n}^{C}(t)\right)\right)\right)} \times \\
& \times \sigma_{n}^{2}\left(X_{n}^{C}(t)\right) \exp \left(-4 \int_{b_{n}}^{X_{n}^{C}(t)} \frac{f_{n}(z)}{\sigma_{n}^{2}(z)} \mathrm{d} z\right) \mathrm{d} t
\end{aligned}
$$

Comparing with (36), we get:

$$
\begin{aligned}
& \mathrm{d} s^{-1}\left(s_{n}\left(X_{n}^{C}(t)\right)\right)=\frac{s_{n}^{\prime}\left(X_{n}^{C}(t)\right) \sigma_{n}\left(X_{n}^{C}(t)\right)}{s^{\prime}\left(s^{-1}\left(s_{n}\left(X_{n}^{C}(t)\right)\right)\right)} \mathrm{d} W(t) \\
& \quad+\frac{s_{n}^{\prime 2}\left(X_{n}^{C}(t)\right) \sigma_{n}^{2}\left(X_{n}^{C}(t)\right)}{\sigma^{2}\left(s^{-1}\left(s_{n}\left(X_{n}^{C}(t)\right)\right)\right) s^{\prime 2}\left(s^{-1}\left(s_{n}\left(X_{n}^{C}(t)\right)\right)\right)} g\left(s^{-1}\left(s_{n}\left(X_{n}^{C}(t)\right)\right)\right) \mathrm{d} t .
\end{aligned}
$$

The rest of the proof follows trivially from this latter formula and (30).

3.6.4. Proof of Lemma 3.14. First, let us show that $\alpha_{n} \rightarrow 0$ uniformly on $\left[x_{1}, x_{2}\right]$. Because $\sigma_{n} \rightarrow \sigma$ uniformly on $\left[x_{1}, x_{2}\right]$, we need only to prove that uniformly on $\left[x_{1}, x_{2}\right]$,

$$
\frac{s_{n}^{\prime}(x)}{s^{\prime}\left(s^{-1}\left(s_{n}(x)\right)\right)} \rightarrow 1
$$

and then to apply Lemma 4.1 (i). Now, let us show (37). First, $s_{n} \rightarrow s$ uniformly on $\left[x_{1}, x_{2}\right]$. Since $s^{-1}$ is continuous in a neighborhood $(s(-\infty), s(\infty))$ of $\left[s\left(x_{1}\right), s\left(x_{2}\right)\right]$, by Lemma 4.1 (iii), $s^{-1}\left(s_{n}(x)\right) \rightarrow s^{-1}(s(x)) \equiv x$ uniformly on $\left[x_{1}, x_{2}\right]$. Applying Lemma 4.1 (iii) again, we get: $s^{\prime}\left(s^{-1}\left(s_{n}(x)\right)\right) \rightarrow s^{\prime}(x)$ uniformly on $\left[x_{1}, x_{2}\right]$. By Lemma 4.1 (ii), uniformly on $\left[x_{1}, x_{2}\right]$ we have:

$$
\frac{1}{s^{\prime}\left(s^{-1}\left(s_{n}(x)\right)\right)} \rightarrow \frac{1}{s^{\prime}(x)}
$$

Finally, $s_{n}^{\prime} \rightarrow s^{\prime}$ uniformly on $\left[x_{1}, x_{2}\right]$. Applying Lemma 4.1 (i) again, we get (37)). This completes the proof that $\alpha_{n} \rightarrow 0$ uniformly on $\left[x_{1}, x_{2}\right]$.

Now, let us show $\beta_{n} \rightarrow 0$ uniformly on $\left[x_{1}, x_{2}\right]$. Earlier, we showed $s^{-1}\left(s_{n}(x)\right) \rightarrow x$ uniformly on $\left[x_{1}, x_{2}\right]$. Since $g$ and $\sigma$ are continuous, by Lemma 4.1 (iii), uniformly on $\left[x_{1}, x_{2}\right]$, we have:

$$
g\left(s^{-1}\left(s_{n}(x)\right)\right) \rightarrow g(x), \quad \sigma\left(s^{-1}\left(s_{n}(x)\right)\right) \rightarrow \sigma(x) .
$$

By Lemma 4.1 (ii), uniformly on $\left[x_{1}, x_{2}\right]$,

$$
\frac{1}{\sigma^{2}\left(s^{-1}\left(s_{n}(x)\right)\right)} \rightarrow \frac{1}{\sigma^{2}(x)}
$$

Next, we can represent the coefficient $\beta_{n}(x)$ as

$$
\beta_{n}(x)=\frac{\left(\alpha_{n}(x)+\sigma_{n}(x)\right)^{2}}{\sigma^{2}\left(s^{-1}\left(s_{n}(x)\right)\right)} g\left(s^{-1}\left(s_{n}(x)\right)\right)-g(x) .
$$

Finally, we use the already proven fact that $\alpha_{n} \rightarrow \alpha$ uniformly on $\left[x_{1}, x_{2}\right]$. The rest of the proof consists of a few more applications of Lemma 4.1 .

3.6.5. Proof of Lemma 3.15. Assume the event $A_{n}(\varepsilon)$ has happened. We have: $t_{1}^{\prime}<T_{C}^{(n)}$, because otherwise $Y_{n}$ would be constant on $\left[t_{1}^{\prime}, t_{2}^{\prime}\right]$. Without loss of generality, we can assume $t_{2}^{\prime} \leq T_{C}^{(n)}$ (otherwise, just substitute $t_{2}^{\prime} \wedge T_{C}^{(n)}$ for $t_{2}^{\prime}$ ). From Lemma 3.13, we get:

$$
Y_{n}\left(t_{2}^{\prime}\right)-Y_{n}\left(t_{1}^{\prime}\right)=\int_{t_{1}^{\prime}}^{t_{2}^{\prime}} \beta_{n}\left(X_{n}(t)\right) \mathrm{d} t+\int_{t_{1}^{\prime}}^{t_{2}^{\prime}} \alpha_{n}\left(X_{n}(t)\right) \mathrm{d} W(t) .
$$


But $X_{n}(t) \in[\eta, C]$ for $t \in\left[t_{1}^{\prime}, t_{2}^{\prime}\right]$, and $\beta_{n} \rightarrow 0$ uniformly on $[\eta, C]$. Find $n_{1}$ such that for all $n \geq n_{1}$ and $x \in[\eta, C]$, we have: $\left|\beta_{n}(x)\right|<\varepsilon /(2 T)$. Then for $n \geq n_{1}$, we get:

$$
\left|\int_{t_{1}^{\prime}}^{t_{2}^{\prime}} \beta_{n}\left(X_{n}(t)\right) \mathrm{d} t\right|<T \cdot \frac{\varepsilon}{2 T}=\frac{\varepsilon}{2}, \quad \text { therefore } \int_{t_{1}^{\prime}}^{t_{2}^{\prime}} \alpha_{n}\left(X_{n}(t)\right) \mathrm{d} W(t)>\varepsilon-\frac{\varepsilon}{2}-\frac{\varepsilon}{2} .
$$

Now, consider the process

$$
M_{n}=\left(M_{n}(t), 0 \leq t \leq T\right), \quad M_{n}(t)=\int_{0}^{t} \alpha_{n}\left(X_{n}(u)\right) \mathrm{d} W(u) .
$$

We have already proved that for $n \geq n_{1}$,

$$
A_{n}(\varepsilon) \subseteq\left\{\exists t_{1}^{\prime}, t_{2}^{\prime} \in[0, T]: M_{n}\left(t_{2}^{\prime}\right)-M_{n}\left(t_{1}^{\prime}\right)>\varepsilon / 2\right\} .
$$

Apply Lemma 4.3 from Appendix to $\delta:=T, K:=\max _{[\eta, C]}\left|\alpha_{n}\right|$ and get: for a constant $\varkappa>0$,

$$
\mathbf{P}\left(\exists t_{1}^{\prime}, t_{2}^{\prime} \in[0, T]: M_{n}\left(t_{2}^{\prime}\right)-M_{n}\left(t_{1}^{\prime}\right)>\varepsilon / 2\right) \leq \varkappa \frac{\left(\max _{[\eta, C]}\left|\alpha_{n}\right|\right)^{4} T}{(\varepsilon / 2)^{2}} T \rightarrow 0,
$$

because $\alpha_{n} \rightarrow 0$ uniformly on $[\eta, C]$. The rest is trivial.

\section{Appendix}

Lemma 4.1. Consider the functions $\varphi, \varphi_{n}, \psi, \psi_{n}: \mathbb{R} \rightarrow \mathbb{R}, n=1,2, \ldots$

(i) Suppose $\varphi_{n} \rightarrow \varphi$ and $\psi_{n} \rightarrow \psi$ uniformly on $\left[x_{1}, x_{2}\right] \subseteq \mathbb{R}$. If $\varphi$ and $\psi$ are continuous on this interval, then $\varphi_{n} \psi_{n} \rightarrow \varphi \psi$ uniformly on $\left[x_{1}, x_{2}\right]$.

(ii) If $\varphi_{n} \rightarrow \varphi$ uniformly on $\left[x_{1}, x_{2}\right]$, and $\varphi$ is continuous and strictly positive on this interval, then $1 / \varphi_{n} \rightarrow 1 / \varphi$ uniformly on $\left[x_{1}, x_{2}\right]$.

(iii) If $\varphi_{n} \rightarrow \varphi$ uniformly on $\left[x_{1}, x_{2}\right]$, and $\psi$ is continuous in a neighborhood of $\left[\varphi\left(x_{1}\right), \varphi\left(x_{2}\right)\right]$, then $\psi\left(\varphi_{n}\right) \rightarrow \psi(\varphi)$ uniformly on $\left[x_{1}, x_{2}\right]$.

(iv) If $\varphi_{n} \rightarrow \varphi$ uniformly on $\left[x_{1}, x_{2}\right], \psi_{n} \rightarrow \psi$ uniformly on $\left[0, x_{0}+\varepsilon\right]$ for some $\varepsilon>0$, and $\varphi(x) \in\left[0, x_{0}\right]$ for $x \in\left[x_{1}, x_{2}\right]$, then $\psi_{n}\left(\varphi_{n}\right) \rightarrow \psi(\varphi)$ uniformly on $\left[x_{1}, x_{2}\right]$.

The proof is trivial and is omitted.

Lemma 4.2. If $x_{n}(t) \rightarrow x(t)$ uniformly on $[0, T]$, and $x(t)$ is a continuous nonnegative function on $[0, T]$, then $\sigma_{n}\left(x_{n}(t)\right) \rightarrow \sigma(x(t))$ uniformly on $[0, T]$.

Proof. Fix $\delta>0$. By condition (v) of Theorem 2.1, there exists $\varepsilon>0$ such that for $y \in(-\varepsilon, 0)$, we have: $\left|\sigma_{n}(y)-\sigma_{n}(0)\right|<\delta, n=1,2, \ldots$ Let $C:=\max _{[0, T]} x(t)$. Because of uniform convergence $x_{n} \rightarrow x$, there exists $n_{0}$ such that for $n>n_{0}$, we have:

$$
x_{n}(t) \in[-\varepsilon, C+\varepsilon], t \in[0, T] .
$$

By condition (iv) of Theorem 2.1, there exists $n_{1}$ such that for $n>n_{1}, y \in[0, C+\varepsilon]$, we have: $\left|\sigma_{n}(y)-\sigma(y)\right|<\delta$. Therefore, for $y \in[-\varepsilon, 0]$ and $n \geq n_{0} \vee n_{1}$, we have:

$$
\left|\sigma_{n}(y)-\sigma(y)\right|=\left|\sigma_{n}(y)-\sigma(0)\right| \leq\left|\sigma_{n}(y)-\sigma_{n}(0)\right|+\left|\sigma_{n}(0)-\sigma(0)\right| \leq \delta+\delta=2 \delta .
$$

Because the function $\sigma$ is continuous on $[-\varepsilon, C+\varepsilon]$, there exists $\eta>0$ such that for $y_{1}, y_{2} \in$ $[-\varepsilon, C+\varepsilon],\left|y_{1}-y_{2}\right|<\eta$, we have: $\left|\sigma\left(y_{1}\right)-\sigma\left(y_{2}\right)\right|<\delta$. Take an $n_{2}$ such that for $t \in[0, T], n \geq n_{2}$, $\left|x_{n}(t)-x(t)\right|<\eta$. Therefore,

$$
\left|\sigma\left(x_{n}(t)\right)-\sigma(x(t))\right|<\delta \text { for } n \geq n_{0} \vee n_{1} \vee n_{2}
$$


From (38) and (39), we get: $\left|\sigma_{n}\left(x_{n}(t)\right)-\sigma(x(t))\right| \leq 2 \delta$. Thus,

$$
\left|\sigma_{n}\left(x_{n}(t)\right)-\sigma(x(t))\right| \leq\left|\sigma_{n}\left(x_{n}(t)\right)-\sigma\left(x_{n}(t)\right)\right|+\left|\sigma\left(x_{n}(t)\right)-\sigma(x(t))\right| \leq 2 \delta+\delta=3 \delta .
$$

Therefore, for every $\delta>0$ we found $N(\delta):=n_{0} \vee n_{1} \vee n_{2}$ such that for every $t \in[0, T]$, we get: $\left|\sigma_{n}\left(x_{n}(t)\right)-\sigma(x(t))\right| \leq 3 \delta$.

Lemma 4.3. Take a real-valued random process $\gamma=(\gamma(t), 0 \leq t \leq T)$ such that $\int_{0}^{T} \gamma^{2}(t) \mathrm{d} t<\infty$ a.s. Let $W=(W(t), 0 \leq t \leq T)$ be a standard Brownian motion. Fix constants $x_{0} \in \mathbb{R}$ and $\delta \in(0, T], K, D>0$. Define a random process $X=(X(t), 0 \leq t \leq T)$ by

$$
X(t)=x_{0}+\int_{0}^{t} \gamma(s) \mathrm{d} W(s), \quad 0 \leq t \leq T .
$$

Take an interval $I:=[\alpha, \beta] \subseteq \mathbb{R}$ with $D \leq \beta-\alpha$. Assume that the process $X$ has the following property: for all $t \in[0, T]$, if $X(t) \in I$, then $|\gamma(t)| \leq K$. Consider the following event:

$$
A:=\left\{\exists t_{1}, t_{2} \in[0, T]: 0 \leq t_{2}-t_{1} \leq \delta, X\left(t_{1}\right), X\left(t_{2}\right) \in I,\left|X\left(t_{2}\right)-X\left(t_{1}\right)\right| \geq D\right\} .
$$

Then

$$
\mathbf{P}(A) \leq \varkappa \frac{T K^{4}}{D^{2}} \delta, \quad \text { for } \varkappa=\frac{8192}{3} .
$$

Proof. Take $M$ to be large enough so that $\delta_{0}:=T / M \leq \delta$, for example $M=\lfloor T / \delta\rfloor+1$. Then $T / \delta \geq 1$, because $\delta \in(0, T]$. Therefore, $M \leq 2 T / \delta$, and $\delta_{0} \leq \delta \leq 2 \delta_{0}$. Now, let $s_{i}:=\delta_{0} i$ for $i=0, \ldots, M$. These points partition the whole time interval $[0, T]$ into $M$ subintervals of equal length $\left[s_{i-1}, s_{i}\right], i=1, \ldots, M$. For each $i=0, \ldots, M-3$, consider the event

$$
A_{i}=\left\{\exists t \in\left[s_{i}, s_{i+3}\right]: X(t), X\left(s_{i}\right) \in I,\left|X(t)-X\left(s_{i}\right)\right| \geq D / 2\right\}
$$

By continuity of $X$, we can also write $A_{i}$ in an equivalent form:

$$
A_{i}=\left\{\exists t \in\left[s_{i}, s_{i+3}\right]: X(t), X\left(s_{i}\right) \in I,\left|X(t)-X\left(s_{i}\right)\right|=D / 2\right\} .
$$

Lemma 4.4. We have:

$$
A \subseteq \bigcup_{i=0}^{M-3} A_{i}
$$

Proof. Suppose the event $A$ has happened. That is, there exist $t_{1}, t_{2} \in[0, T]$ such that $\left|t_{1}-t_{2}\right| \leq \delta$ and $\left|X\left(t_{1}\right)-X\left(t_{2}\right)\right| \geq D$. Then $\left|t_{1}-t_{2}\right| \leq 2 \delta_{0}$. In other words, the two points $t_{1}$ and $t_{2}$ are at a distance no more than twice the length of a small subinterval. Therefore, there exists $i=0, \ldots, M-3$ such that $s_{i} \leq t_{1} \leq t_{2} \leq s_{i+3}$ or $s_{i} \leq t_{2} \leq t_{1} \leq s_{i+3}$, depending on whether $t_{1}$ or $t_{2}$ is greater. Now, we shall prove that the event $A_{i}$ happened. Assume the converse; then

$$
\left|X\left(t_{1}\right)-X\left(s_{i}\right)\right|<\frac{D}{2} \text { and }\left|X\left(t_{2}\right)-X\left(s_{i}\right)\right|<\frac{D}{2} .
$$

Therefore, $\left|X\left(t_{1}\right)-X\left(t_{2}\right)\right| \leq\left|X\left(t_{1}\right)-X\left(s_{i}\right)\right|+\left|X\left(t_{2}\right)-X\left(s_{i}\right)\right|<\frac{D}{2}+\frac{D}{2}=D$. This contradiction completes the proof.

Lemma 4.5. Assume $X(0)=x \in[\alpha+\varepsilon, \beta-\varepsilon]$ for some $\varepsilon>0$. Let

$$
\tau:=\inf \{t \in[0, T]|| X(t)-x \mid=\varepsilon\} \text {. }
$$

Then for $\eta>0$ we have:

$$
\mathbf{P}(\tau \leq \eta) \leq \frac{256}{27} \frac{K^{4} \eta^{2}}{\varepsilon^{2}}
$$


Proof. The process $X(t \wedge \tau)$ is a square-integrable martingale with values in $I=[\alpha, \beta]$. Therefore,

$$
\langle X\rangle_{t \wedge \tau}=\int_{0}^{t \wedge \tau} \gamma^{2}(u) \mathrm{d} u \leq K^{2}(t \wedge \tau) .
$$

Let us make a time-change: for some standard Brownian motion $B=(B(s), s \geq 0)$, we have: $X(t \wedge \tau)=x+B\left(\langle X\rangle_{t \wedge \tau}\right)$, for all $t \geq 0$. Therefore,

$$
\{\tau \leq \eta\}=\{|X(\tau \wedge \eta)-x| \geq \varepsilon\}=\left\{\left|B\left(\langle X\rangle_{\tau \wedge \eta}\right)\right| \geq \varepsilon\right\} \subseteq\left\{\max _{\left[0, K^{2} \eta\right]}|B(s)| \geq \varepsilon\right\} .
$$

By Markov's inequality,

$$
\mathbf{P}\left\{\max _{\left[0, K^{2} \eta\right]}|B(s)| \geq \varepsilon\right\} \leq \frac{\operatorname{E~max}_{\left[0, K^{2} \eta\right]} B(s)^{4}}{\varepsilon^{4}} .
$$

By Doob's martingale inequality and the fact that for $\xi \sim \mathcal{N}\left(0, \sigma^{2}\right)$ we have: $\mathbf{E} \xi^{4}=3 \sigma^{4}$,

$$
\mathbf{E} \max _{\left[0, K^{2} \eta\right]} B(s)^{4} \leq\left(\frac{4}{3}\right)^{4} \mathbf{E}\left[B\left(K^{2} \eta\right)\right]^{4}=\frac{256}{81} \cdot 3\left(K^{2} \eta\right)^{2}=\frac{256}{27} K^{4} \eta^{2} .
$$

Comparing (40), (41) and (42), we complete the proof.

Lemma 4.6. $\mathbf{P}\left(A_{0}\right) \leq \frac{4096}{3} D^{-2} K^{4} \delta^{2}$.

Proof. Case 1: $x=X(0) \in[\alpha+D / 2, \beta-D / 2]$. Apply Lemma 4.5] to $\varepsilon=D / 2$ and $\eta=s_{3}=3 \delta_{0}$. Since $\delta_{0} \leq \delta$, we get:

$$
\mathbf{P}\left(A_{0}\right)=\mathbf{P}\left(\tau \leq s_{3}\right) \leq \frac{256}{27} \frac{9 K^{4} \delta_{0}^{2}}{(D / 2)^{2}}=\frac{1024}{3} \frac{K^{4} \delta_{0}^{2}}{D^{2}} \leq \frac{1024}{3} \frac{K^{4} \delta^{2}}{D^{2}},
$$

Case 2: $x=X(0) \in[\alpha, \alpha+D / 2]$. Then $X(\tau)=x+D / 2$ (because there is not enough "room" in the interval $I=[\alpha, \beta]$ for the process $X$ to go down rather than up). Let

$$
\tau^{\prime}:=\inf \{t \geq 0 \mid X(t)=x+D / 4\} .
$$

Then $X\left(\tau^{\prime}\right) \in[\alpha+D / 4, \beta-D / 4]$, because $D \leq \beta-\alpha$. Apply Lemma 4.5 to $X(t)-X\left(\tau^{\prime}\right)$ instead of $X$, to $\varepsilon=D / 4$ and $\eta=s_{3}$. Then we get:

$$
\mathbf{P}\left(A_{0}\right) \leq \frac{256}{27} \frac{9 K^{4} \delta_{0}^{2}}{(D / 4)^{2}} \leq \frac{4096}{3} \frac{K^{4} \delta^{2}}{D^{2}} .
$$

Case 3: $x=X(0) \in[\beta-D / 2, \beta]$. This case is analogous to Case 2 .

Similarly, we can show that for $i=0, \ldots, M-3$,

$$
\mathbf{P}\left(A_{i}\right) \leq \frac{4096}{3} \frac{K^{4} \delta^{2}}{D^{2}} .
$$

Applying Lemma 4.4, we get:

$$
\mathbf{P}(A) \leq \sum_{i=0}^{M-3} \mathbf{P}\left(A_{i}\right) \leq M \frac{4096}{3} \frac{K^{4} \delta^{2}}{D^{2}} .
$$

But $\delta \leq T$, and so $M \leq 2 T / \delta$. The rest of the proof is trivial.

\section{ACKNOWELDGEMENTS}

The authors would like to thank Ioannis Karatzas, Mykhaylo Shkolnikov, Leszek Slominski, and Ruth Williams for help and useful discussion. This research was partially supported by NSF grants DMS 1007563, DMS 1308340, DMS 1405210, and DMS 1409434. 


\section{REFERENCES}

[1] Richard F. Bass, Pei Hsu (1991). Some Potential Theory for Reflecting Brownian Motion in Holder and Lipschitz Domains. Ann. Probab. 19 (2), 486-508.

[2] Patrick Billingsley (1999). Convergence of Probability Measures. Jonh Wiley \& Sons, 2nd edition.

[3] Emmanuel Cepa (1998). Problame de Skorohod Multivoque. Ann. Probab. 26 (2), 500-532.

[4] Nicole El Karoui, Christophe Kapoudjian, Etienne Pardoux, Shi Ge Peng, Marie-Claire Quenez (1997). Reflected Solutions of Backward SDEs and Related Obstacle Problems for PDEs. Ann. Probab. 25 (2), 702-737.

[5] Masatoshi Fukushima, Matsyuo Tomisaki (1996). Construction and Decomposition of Reflecting Diffusions on Lipschitz Domains with Holder Cusps. Probab. Th. Rel. Fields 106 (4), 521-557.

[6] I.I. Ginman, A. V. Skorohod (1972). Stochastic Differential Equations. Springer-Verlag. Ergebnisse der Mathematik und ihrer Grenzgebiete, 72.

[7] J. Michael Harrison, I. Marting Reiman (1981). Reflected Brownian Motion on an Orthant. Ann. Probab. 9 (2), 302-308.

[8] J. Michael Harrison, Ruth J. Williams (1987). Brownian Models of Open Queueing Networks with Homogeneous Customer Populations. Stochastics, 22 (2), 77-115.

[9] J. Michael Harrison, Ruth J. Williams (1987). Multidimensional Reflected Brownian Motions Having Exponential Stationary Distributions. Ann. Probab. 15 (1), 115-137.

[10] Tomasz Klimsiak, Andrzej Rozkosz, Leszek Slominski (2015). Reflected BSDEs in Time-Dependent Convex Regions. Stoch. Proc. Appl. 125 (2), 571-596.

[11] Weronika Laukajtys, Leszek Slominski (2003). Penalization Methods for Reflecting Stochastic Differential Equations with Jumps. Stoch. Stoch. Rep. 75 (5), 275-293.

[12] Weronika Laukajtys, Leszek Slominski (2013). Penalization Methods for the Skorokhod Problem and Reflecting Stochastic Differential Equations with Jumps. Bernoulli 19 (5A), 1750-1775.

[13] Antoine Lejay (2006). On the Constructions of the Skew Brownian Motion. Probab. Surv. 3, 413-466.

[14] Pierre-Louis Lions, Alain-Sol Sznitman (1984). Stochastic Differential Equations with Reflecting Boundary Conditions. Comm. Pure Appl. Math. 37 (4), 511-537.

[15] Pierre-Louis Lions, Jose Luis Menaldi, Alain-Sol Sznitman (1981). Construction de de Processus de Diffusion Reflechis par Penalisation du Domaine. C.R. Acad. Sci. Paris Ser. I Math. 292, 559-562.

[16] Lucian Maticiuc, Aurel Rascanu, Leszek Slominski, Mateusz Topolevski (2015). Cadlag Skorokhod Problem Driven by a Maximal Monotone Operator. J. Math. Anal. Appl. 429 (2), 1305-1346.

[17] Henry P. McKean JR. (1963). Skorohod's Stochastic Integral Equation for a Reflecting Barrier Diffusion. J. Math. Kyoto Univ. 3, 85-88.

[18] Jose-Luis Menaldi, Maurice Robin (1985). Reflected Diffusion Processes with Jumps. Ann. Probab. 13 (2), 319-341.

[19] Jose-Luis Menaldi, Maurice Robin (2005). Construction and Control of Reflected Diffusion with Jumps. Lecture Notes in Control and Information Sciences 69 309-322.

[20] Neil O' Connell, Janosch Ortmann (2014). Product-Form Invariant Measures for Brownian Motion with Drift Satisfying a Skew-Symmetry Condition. Lat. Am. J. Probab. Math. Stat. 11 (1), 307-329.

[21] Etienne Pardoux, Ruth J. Williams (1994). Symmetric Reflected Diffusions. Ann. Inst. H. Poincare Probab. Stat. 30 (1), 13-62.

[22] Nikolai Portenko (1979). Diffusion Processes with Generalized Drift Coefficients. Th. Probab. Appl. 24 (1), 62-78.

[23] Nikolai Portenko (1980). Stochastic Differential Equations with Generalized Drift Vector. Th. Probab. Appl. 24 (2), 338-353.

[24] Andrzej Rozkosz, Leszek Slominski (2012). Lp Solutions of Reflected BSDEs Under Monotonicity Condition. Stoch. Proc. Appl. 122 (12), 3875-3900.

[25] Andrey Sarantsev (2015). Weak Approximation of Multidimensional Obliquely Reflected Brownian Motion by Solutions of SDE. Available at arXiv:1509.01777.

[26] V. A. Shalaumov (1980). On the Behavior of a Diffusion Process with a Large Drift Coefficient in a Halfspace. Th. Probab. Appl. 24 (3), 592-598.

[27] A. V. Skorohod (1961). Stochastic Equations for Diffusion Processes in a Bounded Region. Th. Probab. Appl. 6 (3), 264-274.

[28] A. V. Skorohod (1962). Stochastic Equations for Diffusion Processes in a Bounded Region. Th. Probab. Appl. 7 (1), 3-23. 
[29] Leszek Slominski (2013). Weak and Strong Approximations of Reflected Diffusions via Penalization Methods. Stoch. Proc. Appl. 123 (3), 752-763.

[30] Daniel W. Stroock, S. R. Srinivasa Varadhan (1971). Diffusion Processes with Boundary Conditions. Comm. Pure Appl. Math. 24 (2), 147-225.

[31] Hiroshi Tanaka (1979). Stochastic Differential Equations with Reflecting Boundary Conditions in Convex Regions. Hiroshima Math. J. 9 (1), 163-177.

[32] Shinzo Watanabe (1971). On Stochastic Differential Equations for Multidimensional Diffusion Processes with Boundary Conditions. J. Math. Kyoto Univ. 11 (1), 169-180.

[33] Shinzo Watanabe (1971). On Stochastic Differential Equations for Multidimensional Diffusion Processes with Boundary Conditions II. J. Math. Kyoto Univ. 11 (3), 545-551.

[34] Ruth J. Williams (1995). Semimartingale Reflecting Brownian Motions in the Orthant. Stochastic Networks, IMA Vol. Math. Appl. 71, 125-137.

[35] Ruth J. Williams, W. A. Zheng (1990). On Reflecting Brownian Motion - a Weak Convergence Approach. Ann. Inst. H. Poincare Probab. Stat. 26 (3), 461-488.

Department of Mathematics, Columbia University

E-mail address: cam.bruggeman@gmail.com

Department of Statistics and Applied Probability, University of California, Santa Barbara

E-mail address: sarantsev@pstat.ucsb.edu 